\title{
Intramolecular Sulfamylation Reaction of $N$-(1H-indol-3-yl)ethylsulfamides: Synthesis of 2,3,4,9-Tetrahydro-1,2-thiazino[5,6-b]indole 1,1-Dioxides
}

\author{
Ji Sun Lee, Dong Geol Lee, Dong Seub Park, Sun Hee Kim, Han Sik Yoon, ${ }^{*}$ and Chai-Ho Lee \\ Department of Chemistry. Wonkwang Liniversity, /ksan, Jeonbuk 570-7t9, Korea \\ "School of Medical Radiation. Wonkwang Helth Science College, Jeonbuk 570-750, Korea \\ Received Inthe 26, 2003
}

Key Words : Sulfamylation reaction, Indole, Sulfamide, 1,2-Thiazinol5,6-b]indole 1,1-dioxides

The pharmacological properties of sulfamides have commanded the interest of organic and medicinal chemists. The need for additional information is further magnified by the many useful biological properties (i.e., anticonvulsant, hyperglycemic, antihypertensive, histamine $\mathrm{H}_{2}$-receptor antagonist, herbicidal, HCMV inhibitor) that have been observed for sulfamide-containing compounds. 'The synthesis and reaction of sulfamides have been considered several times in reviews which were partially or completely devoted to sufamides, ${ }^{2}$ One of the earliest known reactions of sulfamide is its ability to produce substituted sulfamides with alkylamines. ${ }^{3}$ The reaction of sulfamide with aromatic anines yields not only diarylsulfamides but also gives rise to rearranged sulfanilanilides. The reaction of $N, N^{\prime}$-dialkylsulfamides with hypochloridate and base leads to the formation of azoalkanes. ${ }^{5}$ Sulfarnides are not as strong nucleophiles as amines; nevertheless, they can react with electrophilic reagents (i.e. carbonyl reagents, nitriles, and alkyl halides), ti.z.6 Previousely, we have demonstrated general route for the synthesis of the 1,2,5-thiadiazolidine 1,1-dioxides ${ }^{\bar{\gamma}}$ and $\alpha$-sulfamidoalkylation transformations from arylalkylsulfamides for the preparation of sulfamide derivatives. ${ }^{\text {oli. }} 8$

In the present study, we report on the intramolecular sulfamylation reaction of $N$-(1/I-indol-3-yl)ethylsulfamides
2 for the generation of 2,3,4,9-tetrahydrothiazino[5,6-b]indoles 3 (Scheme 1).

The starting sulfamides $\mathbf{2} \mathbf{a}$ and $\mathbf{b}$ were prepared from the treatment of sulfamide with the corresponding 2-arylethylamines $\mathrm{Ia}$ and $\mathbf{b}$ at reflux for $12 \mathrm{~h}$ in $\mathrm{H}_{2} \mathrm{O}$, according to established synthetic protocols. "When $t$-butanol was reacted with an equimolar quantity of chlorosulfonylisocyanate $\left(\mathrm{OCNSO} \mathrm{Sl}_{2} \mathrm{Cl}\right.$ in chloroform, followed by reaction with amine $1 \mathrm{c}$, the resultant was hydrolyzed with trifluoroacetic acid to give sulfamide 2 c. $^{10}$ Treatment of sulfamides 2 at reflux in acetic acid produced thiazinoindoles 3 as the major product $(51-55 \%)$. A key process is the intramolecular sulfamylation reaction $(2 \rightarrow 3)$, which is considered to involve intramolecular aromatic attack of indole ring on protonated sulfamide group of 2 (Scheme 1). ${ }^{1}$ Compounds 3 have been assigned as 2,3,4,9-tetrahydro-1,2-thiazino[5,6b]indole 1,1-dioxide on the basis of the ${ }^{1} \mathrm{H}$ - and ${ }^{13} \mathrm{C}$-NMR (500 MHz) spectral data, and mass spectroscopy. Distinctive signals of $3 \mathbf{a}$ and $\mathbf{b}$ were noted in ${ }^{2} \mathrm{H}$ NMR spectra for the methylene resonances at $\mathrm{C}-4(\delta 2.87-2.95)$ and $\mathrm{C}-3(\delta 3.63-$ $3.82)$ and in the ${ }^{1.5} \mathrm{C}$ NMR spectra for the $\mathrm{C}-4(\delta 22.2-22.3)$ and $\mathrm{C}-3(\delta 43.8-43.9 \mathrm{ppm})$. Key signals of $3 \mathbf{c}$ detected in ${ }^{~} \mathrm{H}$ NMR spectra for methylene resonances at $\mathrm{C}-4(\delta, 3.14$ and $3.38)$ and $C-3(\delta 4.74 \mathrm{ppm})$ and in the ${ }^{13} \mathrm{C}$ NMR spectra for the $\mathrm{C}-4(\delta 25.6)$ and $\mathrm{C}-3(\delta 52.1 \mathrm{ppm})$. Additional evidence

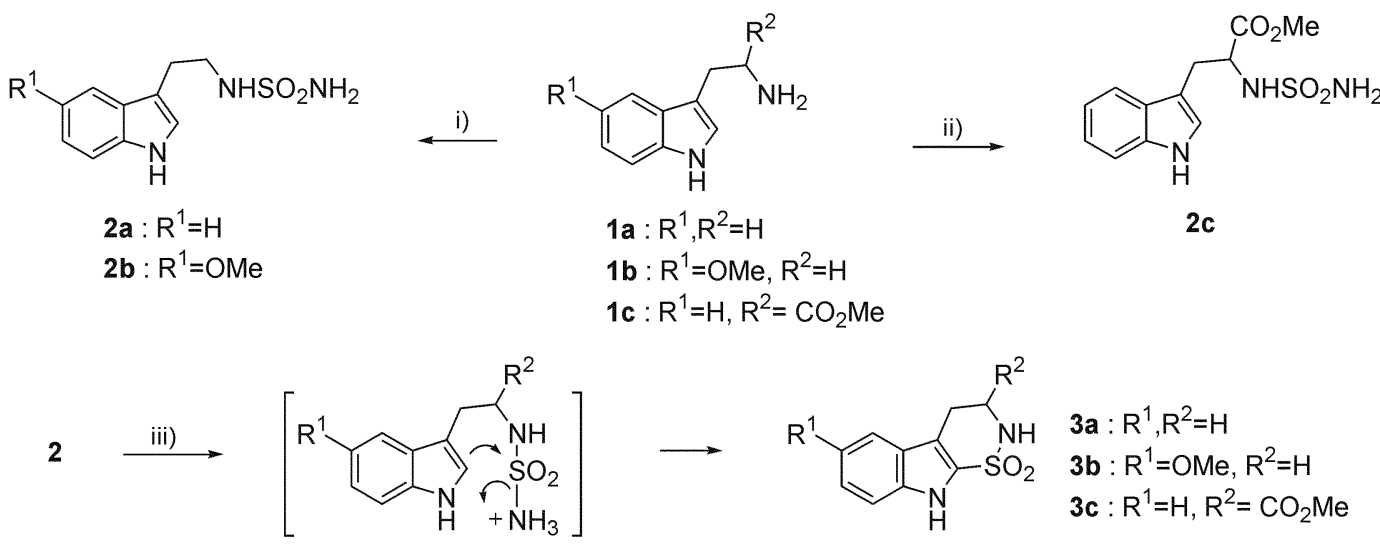

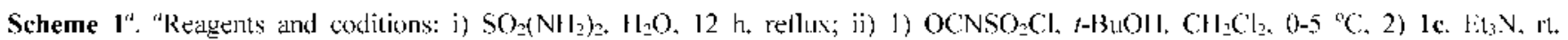
3) $\mathrm{Cl}_{2} \mathrm{CO}_{2} \mathrm{II}$; iii) $\mathrm{AcOll}$. 12 h. reflux.

Corresponding author. E-mail: chaiho(e)wonkwang.ac.kr 
Table 1. Crystal data and strueture refinement for $3 \mathbf{a}$

\begin{tabular}{|c|c|}
\hline Fimpirical formula & $\mathrm{C}_{10} \mathrm{Il}_{10} \mathrm{~N}_{2} \mathrm{O}_{2} \mathrm{~S}$ \\
\hline Fontula weight & 222.26 \\
\hline lemperature & $293(2) \mathrm{K}$ \\
\hline Wavelength & $0.71070 \mathrm{~A}$ \\
\hline Cnystal system. space group & Monoclinic. $\mathrm{P}_{2} \mathrm{ic}$ \\
\hline \multirow[t]{3}{*}{ Unit cell dimensions } & $a=10.2820(8) \mathrm{A}$ \\
\hline & $b=10.3452(6) \lambda . \beta=116.027(8)^{\circ}$ \\
\hline & $c=10.4122(14) \lambda$ \\
\hline Volume & $995.22(17) \Lambda^{3}$ \\
\hline \%. D $\mathrm{D}_{\mathrm{calt}}$ & 4. $1.483 \mathrm{~g} / \mathrm{cm}^{3}$ \\
\hline$\mu$ & $0.304 \mathrm{~mm}^{-1}$ \\
\hline$F(000)$ & 464 \\
\hline Crystal size & $0.5 \times 0.5 \times 0.5 \mathrm{~mm}$ \\
\hline$\theta$ range for dalla collection & $2.201025 .97^{\circ}$ \\
\hline hkl collected & $112.12 .+12$ \\
\hline Reflections colloeted/unique & $2062 / 1954[\mathrm{R}(\mathrm{int})=0.0587]$ \\
\hline Completeness to $2 \theta=51.94$ & $94.5 \%$ \\
\hline Refinement method & Full-matrix least-squares on $F^{2}$ \\
\hline Data/restraints/parameters & $1954 / 0 / 137$ \\
\hline Goodness-of-fit on $F^{2}$ & 1.048 \\
\hline Final $\mathrm{R}$ indices $|/>2 \sigma(I)|$ & ${ }^{a} R_{1}=0.0516 .{ }^{b} 11 R_{2}=0.1399$ \\
\hline $\mathrm{R}$ indices (all data) & ${ }^{a} R_{1}=0.0703 .{ }^{b}{ }_{11} R_{2}=0.1525$ \\
\hline I:xlinction coeslicient & $0.014(4)$ \\
\hline I argest ditl. peak and hole & 0.602 and -0.621 e. $\dot{\lambda}^{-3}$ \\
\hline
\end{tabular}

for the structure of target compound 3a was provided by a determination of the crystal structure by $\mathrm{X}$-ray diffraction methods. Suitable crystal for X-ray analysis of $\mathbf{3 a}$ has been

Table 2. F.ected Bond lengths $[\dot{A}]$ and Bond Angles (deg) lor Compound 3a

\begin{tabular}{llll}
\hline Bond lengths & & & \\
$S(1)-O(1)$ & $1.431(2)$ & $S(1)-O(2)$ & $1.434(2)$ \\
$S(1)-N(2)$ & $1.613(2)$ & $S(1)-C(1)$ & $1.733(3)$ \\
$V(1)-C(8)$ & $1.371(3)$ & $N(1)-C(1)$ & $1.377(3)$ \\
$V(2)-C(10)$ & $1.478(4)$ & $C(1)-C(2)$ & $1.363(4)$ \\
$C(2)-C(3)$ & $1.433(4)$ & $C(2)-C(9)$ & $1.497(4)$ \\
$C(3)-C(8)$ & $1.406(4)$ & $C(3)-C(4)$ & $1.408(4)$ \\
$C(4)-C(5)$ & $1.377(5)$ & $C(5)-C(6)$ & $1.392(6)$ \\
$C(6)-C(7)$ & $1.371(5)$ & $C(7)-C(8)$ & $1.403(4)$ \\
$C(9)-C(10)$ & $1.515(4)$ & & \\
$130 n d A n g l e s$ & & & \\
$O(1)-S(1)-C(2)$ & $117.25(14)$ & $O(1)-S(1)-N(2)$ & $109.90(13)$ \\
$O(2)-S(1)-V(2)$ & $107.15(13)$ & $O(1)-S(1)-C(1)$ & $109.40(13)$ \\
$O(2)-S(1)-C(1)$ & $110.31(13)$ & $N(2)-S(1)-C(1)$ & $101.67(12)$ \\
$C(8)-N(1)-C(1)$ & $107.3(2)$ & $C(10)-N(2)-S(1)$ & $115.61(19)$ \\
$C(2)-C(1)-V(1)$ & $111.5(2)$ & $C(2)-C(1)-S(1)$ & $123.5(2)$ \\
$V(1)-C(1)-S(1)$ & $124.91(19)$ & $C(1)-C(2)-C(3)$ & $105.5(2)$ \\
$C(1)-C(2)-C(9)$ & $124.8(3)$ & $C(3)-C(2)-C(9)$ & $129.7(3)$ \\
$C(8)-C(3)-C(4)$ & $119.0(3)$ & $C(8)-C(3)-C(2)$ & $107.2(2)$ \\
$C(4)-C(3)-C(2)$ & $133.8(3)$ & $C(5)-C(4)-C(3)$ & $118.5(3)$ \\
$C(4)-C(5)-C(6)$ & $121.2(3)$ & $C(7)-C(6)-C(5)$ & $122.3(3)$ \\
$C(6)-C(7)-C(8)$ & $116.6(3)$ & $N(1)-C(8)-C(7)$ & $129.2(3)$ \\
$V(1)-C(8)-C(3)$ & $108.5(2)$ & $C(7)-C(8)-C(3)$ & $122.3(3)$ \\
$C(2)-C(9)-C(10)$ & $111.2(2)$ & $N(2)-C(1)-C(9)$ & $112.2(2)$ \\
\hline & & &
\end{tabular}

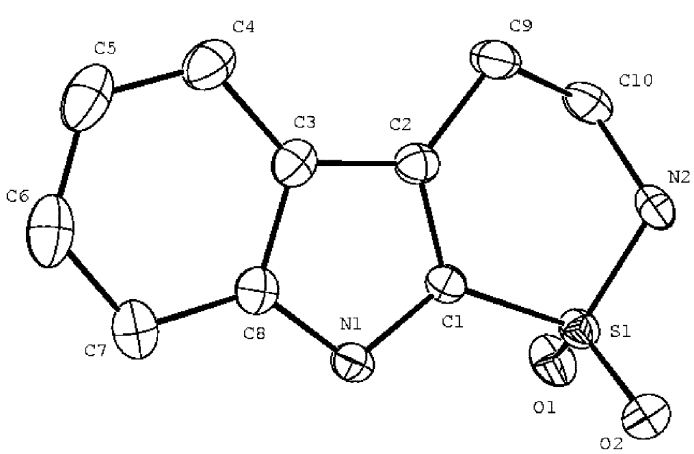

Figure 1. An ORTLP drawing of compound 3a with atomic numbering schems.

obtained in a chloroform solution, and the crystal structures of the compound was determined by X-ray diffraction. Crystal data for complex $\mathbf{3 a}$ are summarized in Table I, refinement details are discussed in the experimental section, and selected bond distances and angles are collected in Table 2. The molecular geometries and atom-labeling schemes are shown in Figure 1.

In conclusion, we have elucidated an intramolecular sulfamylation reaction of $N$-(1H-indol-3-yl)ethylsulfamides for the generation of 2,3,4,9-tetrahydro-1,2-thiazino[5,6b]indole l, I-dioxides.

\section{Experimental Section}

$\mathrm{N}$-|2-(1 H-lndol-3-yl)|ethylsulfamide (2a). A water solution containing of tryptamine 1 a $(1.6 \mathrm{~g} .10 \mathrm{mmol})$ and sulfamide $(1.0 \mathrm{~g}, 10 \mathrm{mmol})$ was heated at reflux for $12 \mathrm{~h}$ and then cooled to room temperature. The solid that precipitated was filtered and then washed with aqueous $1 \mathrm{~N} \mathrm{HCl}$ solution $(20 \mathrm{~mL})$ and water $(3 \times 20 \mathrm{~mL})$ to give the pale yellow powder 1.1 g $\left(49.6 \%\right.$ ) of $2 \mathbf{2}$; $\mathrm{mp} 137-138{ }^{\circ} \mathrm{C}$; IR (KBr) $3422,3420,3400,3264,1321,1140 \mathrm{~cm}^{-1}$; 'H NMR (DMSO-d 6 ) $\delta 2.89$ (t. $J=7.3 \mathrm{~Hz}, 2 \mathrm{H}$ ), 3.14 (q. $J=7.3 \mathrm{~Hz}$. $2 \mathrm{H}), 6.54(\mathrm{~s}, 2 \mathrm{H}), 6.56(\mathrm{t}, J-5.6 \mathrm{~Hz}, \mathrm{lH}), 6.98(\mathrm{td}, J-7.8$ and $0.9 \mathrm{~Hz}, \mathrm{lH}), 7.70(\mathrm{td}, J-7.8$ and $0.9 \mathrm{~Hz}, 1 \mathrm{H}), 7.16(\mathrm{~d} . J$ $-1.9 \mathrm{~Hz}, 1 \mathrm{H}), 7.33(\mathrm{~d}, J-7.8 \mathrm{~Hz}, \mathrm{lH}), 7.5 \mathrm{I}(\mathrm{d}, J-7.8 \mathrm{~Hz}$, $1 \mathrm{H}), 10.8$ (s, NH) ppm; ${ }^{13} \mathrm{C}$ NMR (DMSO-d 6 ) $\delta 25.9 .44 .1$, $111.9,112.2,118.8,121.5,123.3,127.7,136.8 \mathrm{ppm}$; LR FAB MS: calcd for [M-1] 238.3 , found 239.07 .

$\boldsymbol{N}$-|2-(5-Methoxy-1 $\boldsymbol{H}$-indol-3-yl)|ethylsulfamide (2b). The procedure described for the preparation of 2a was employed using $1 \mathrm{~b}(1.9 \mathrm{~g}, 10 \mathrm{mmnol})$ and sulfamide $(1.0 \mathrm{~g}$, $10 \mathrm{mmol}$ ). After workup, $2 \mathrm{~b}$ was obtained in $43.0 \%$ yield (1.2 g); mp 130-132 ${ }^{\circ} \mathrm{C}: \mathrm{IR}(\mathrm{KBr}) 3404,3322,3246,3129$, $1335.1148 \mathrm{~cm}^{-1} ;{ }^{1} \mathrm{H}$ NMR (Acetone-d $\left.{ }_{6}\right) \delta 3.0 \mathrm{l}(\mathrm{t}, J-7.4$ $\mathrm{Hz}, 2 \mathrm{H}), 3.56$ (q. $J-7.4 \mathrm{~Hz}, 2 \mathrm{H}), 3.76(\mathrm{~s}, 3 \mathrm{H}), 5.6 \mathrm{I}(\mathrm{s}, \mathrm{IH})$, $5.88(\mathrm{~s}, 2 \mathrm{H}), 6.75(\mathrm{dd}, J-8.7$ and $2.3 \mathrm{~Hz}, 1 \mathrm{H}), 7.09(\mathrm{~d}, J-$ $2.3 \mathrm{~Hz}, \mathrm{IH}), 7.16(\mathrm{~d}, J-2.3 \mathrm{~Hz}, \mathrm{IH}), 7.26(\mathrm{~d} . J-8.7 \mathrm{~Hz}$, 1H), $9.88(\mathrm{~s}, 1 \mathrm{H}) \mathrm{ppm} ;{ }^{1.9} \mathrm{C}$ NMR (Acetone-d $\left.\mathrm{d}_{6}\right) \delta 25.6,43.9$, 55.1, 100.4, 1II.6, 111.9, 123.2, 123.3, 128.0, 131.9, 153.9 ppm; LR FAB MS: calcd for [M- $]^{-} 268.4$, found 269.08 .

$\mathrm{N}$-|l-Methoxycarbonyl-2-( $\mathbf{H}$-indol-3-yl)|ethylsulfamide (2c). Chlorosulfonylisocyanate $(1.4 \mathrm{~g}, 10 \mathrm{mmol})$ of was 
added dropwise to a cold solution of $t$-butyl alcohol $(0.7 \mathrm{~g}$. $10 \mathrm{mmmol})$ in anhydrous dichloromethane $(10 \mathrm{~mL})$. Then $1 \mathrm{c}$ $(2.2 \mathrm{~g} .10 \mathrm{mmol})$ and trietlyy lanine $(1.2 \mathrm{~g}, 12 \mathrm{nmol})$ was added. The mixture was stirred for $3 \mathrm{~h}$ at room temperature and then washed with $1 \mathrm{~N} \mathrm{HCl}$ and with water several times. The organic layer was concentrated to dryness in wacto. The residue was added to a dichloromethane $(12 \mathrm{~mL})$ solution containing trifluoroacetic acid ( $8 \mathrm{~mL})$, and then the solution was stirred at room temperature for $6 \mathrm{~h}$. The solution was washed with water, dried (anhydrous $\mathrm{MgSO}_{4}$ ) and concentrated in vacto to give $2 \mathrm{c}(2.4 \mathrm{~g} .80 .1 \%$ ): IR (KBr) 3400 . 3261, 3153. 3096. 1341. $1163 \mathrm{~cm}^{-1}$. ${ }^{1} \mathrm{H}$ NMR (Acetone-d $\delta 3.26(\mathrm{~d}, J=6.4 \mathrm{~Hz} .2 \mathrm{H}), 3.60(\mathrm{~s}, 3 \mathrm{H}), 4.35(\mathrm{td}, J=6.4$ and $7.6 \mathrm{~Hz} . \mathrm{lH}) .5 .98(\mathrm{~d} . J=7.6 \mathrm{~Hz} . \mathrm{IH}) .5 .99(\mathrm{~s}, 2 \mathrm{H}), 7.0 \mathrm{l}(\mathrm{td} . J$ $=6.9$ and $0.9 \mathrm{~Hz} . \mathrm{IH}) .7 .09(\operatorname{td} . J=6.9$ and $0.9 \mathrm{~Hz}, \mathrm{IH}) .7 .22$ (d. $J=2.3 \mathrm{~Hz}, 1 \mathrm{H}$ ). 7.37 (d. $J=6.9 \mathrm{~Hz} . \mathrm{lH}$ ). 7.57 (d. $J=6.9$ $\mathrm{Hz}, \mathrm{IH}), 10.08(\mathrm{~s}, \mathrm{IH}) \mathrm{ppm} ;{ }^{13} \mathrm{C}$ NMR (Acetone-d $\left.\mathrm{d}_{6}\right) \delta 28.7$. 51.6, 57.0. 109.4, 111.4, 118.4, 118.8, 121.4. 123.9. 127.7. 136.7. $172.6 \mathrm{ppm}$; LR FAB MS: calcd for $[\mathrm{M}-1]^{-} 296.4$. found 297.08 .

General procedure for intramolecular sulfamylation reaction of 3 . A acetic acid $(20 \mathrm{~mL})$ solution of sulfanides 2 $(5.0 \mathrm{mmol})$ was stirred at reflux for $12 \mathrm{~h}$ and then cooled to rt. The solution was quenched with excess water $(50 \mathrm{~mL})$ and extracted with ethyl acetate $(3 \times 10 \mathrm{~mL})$. The solution was washed with aqueous $5 \% \mathrm{NaHCO}_{3}(20 \mathrm{~mL})$ solution and with water $(3 \times 20 \mathrm{~mL}$ ). and then dried (anhydrous $\mathrm{MgSO}_{4}$ ) and evaporated in vono. The solid was recrystallized from ethyl acetate to give the desired products 3 .

2,3,4,9-Tetrahydro-1,2-thiazino[5,6-b]indole 1,1-dioxide (3a): Compound 3a was obtained from $2 \mathrm{a}(1.2 \mathrm{~g})$ in $53.0 \%$ yield $(0.6 \mathrm{~g})$ : mp $200-245^{\circ} \mathrm{C}$ dec; $\mathrm{IR}(\mathrm{KBr}) 3324.3239$. $1320,1157 \mathrm{~cm}^{-1}$; ${ }^{1} \mathrm{H}$ NMR (DMSO-d $) \delta 2.87$ (t. $J=5.5 \mathrm{~Hz}$. $2 \mathrm{H}), 3.63$ (q. $J=5.5 \mathrm{~Hz} .2 \mathrm{H}$ ). 7.12 (t. $J=7.8 \mathrm{~Hz}, \mathrm{IH}), 7.30$ $(\mathrm{t}, J=7.8 \mathrm{~Hz} . \mathrm{lH}) .7 .4 \mathrm{l}(\mathrm{d}, J=7.8 \mathrm{~Hz}, 1 \mathrm{H}), 7.60$ (d. $J=7.8$ $\mathrm{Hz}, 1 \mathrm{H}$ ), 12.16 (s. 1H) ppm; ${ }^{13} \mathrm{C}$ NMR (DMSO-d $) \delta 22.2$. $43.9,112.9,116.8,120.6 .120 .9 .125 .1,125.5,130.5 .136 .0$ ppin; LR FAB MS: calcd for [M-1] 221.2. found 222.05 .

6-Methoxy-2,3,4,9-tetrahydro-1,2-thiazino[5,6-b]indole 1,1-dioxide (3b): Compound 3b was obtained from $2 \mathrm{~b}$ (1.2 g) in $55.4 \%$ yield $(0.7 \mathrm{~g})$ : $\mathrm{mp} 134-146^{\circ} \mathrm{C}$ dec: IR $(\mathrm{KBr})$ 3291, 3275. 1318, $1154 \mathrm{~cm}^{-1}:{ }^{1} \mathrm{H}$ NMR (Acetone-d $) \delta 2.95$ (t. $J=6.0 \mathrm{~Hz}, 2 \mathrm{H}) \cdot 3.77-3.82(\mathrm{~m} .2 \mathrm{H}) .3 .81$ (s. $3 \mathrm{H}) .6 .44$ (t. $J$ $=7.3 \mathrm{~Hz}, 1 \mathrm{H}), 6.96(\mathrm{dd} . J=2.3$ and $8.7 \mathrm{~Hz}, 1 \mathrm{H}) .7 .07(\mathrm{~d} . J=$ $2.3 \mathrm{~Hz} .1 \mathrm{H}), 7.40(\mathrm{~d} J=8.7 \mathrm{~Hz} .1 \mathrm{H}), 10.93(\mathrm{~s} .1 \mathrm{H}) \mathrm{ppm} \cdot{ }^{13} \mathrm{C}$ NMR (Acetone-d f $\delta 22.3 .43 .8,55.1 .101 .1 .113 .2,116.1$. 116.3. 125.6. 131.0. 131.1. 154.8 ppm: LR FAB MS: calcd for $[M-1]^{-} 251.3$, found 252.06 .

3-Methoxycarbonyl-2,3,4,9-tetrahydro-1,2-thiazino[5,6b]indole 1,1-dioxide (3c): Beginning with sulfamide $2 \mathrm{c}$ (1.5 g). compound $3 \mathrm{c}$ was obtained in $51.2 \%$ yield $(0.7 \mathrm{~g}): \mathrm{mp}$ $170-180^{\circ} \mathrm{C}$ dec $:$ IR $(\mathrm{KBr}) 3314,1744,1341,1165 \mathrm{~cm}^{-1}:{ }^{1} \mathrm{H}$ NMR (Acetone-d ${ }_{6}$ ) $\delta 3.14$ (dd $J=16.8$ and $11.9 \mathrm{~Hz}, 1 \mathrm{H}$ ). 3.38 (dd $J=16.8$ and $4.3 \mathrm{~Hz}, 1 \mathrm{H}$ ), 3.83 (s. $3 \mathrm{H}$ ) 4.74 (ddd. $J$ $=12.3 .11 .9$, and $4.3 \mathrm{~Hz}, 1 \mathrm{H}), 6.91(\mathrm{~d} . J=12.3 \mathrm{~Hz}, 1 \mathrm{H}) .7 .17$ (td. $J=0.9$ and $8.2 \mathrm{~Hz}, 1 \mathrm{H}) .7 .34(\mathrm{td} . J=0.9$ and $8.2 \mathrm{~Hz}$. $1 \mathrm{H}) .7 .52(\mathrm{~d} . J=8.2 \mathrm{~Hz} .1 \mathrm{H}) .7 .67(\mathrm{~d} . J=8.2 \mathrm{~Hz} .1 \mathrm{H}) .11 .23$ (s, IH) ppm; ${ }^{12} \mathrm{C}$ NMR (Acetone-d 6 ) $\delta$ 25.6, 52.1. 56.8 , $112.5,115.4$. 120.3. 120.6. 125.0. 125.4. 130.4. 136.4, 169.5 ppm: LR FAB MS: calcd for [M-1] 279.4 . found 280.05 .

$X$-ray analysis of 3a. Details of the crystal data and summary of intensity data collection parameters for $\mathbf{3 a}$ are given in Table 1. Crystals were grown from chloroform solution stored at room temperature. Crystal was mounted on glass fibers in random orientations, and the data were collected on a Euraf-Nonius CAD4 diffractometer equipped with graphite-monochromated Mo-K $\alpha$ radiation $(\alpha=0.71070$ $\AA$ ) at room temperature. Unit cell parameters were determined by using searcli. center. index and lest-square routine. Structure was solved by the application of direct methods using the SHELX-86 program ${ }^{11}$ and least-squares refinement using SHELEX-97. ${ }^{12}$ Anisotropic thermal parameters were used for all atoms except lydrogen. All the remaining hydrogen atoms were included in calculated positions.

Acknowledgement. This paper was supported by Wonkwang University in 2002.

Supplementary material. Crystallographic Data for the structure reported here have been deposited with the Cambridge Crystallographic Data Centre (Deposition No. CCDC-216058). That data can be obtained free of charge via htp wuw:calcamacukpticatreqcgi (or from the CCDC, 12 Union Road. Cambridge CB2 IEZ, UK: fax: +44 1223 336033: e-mail: depositaccdc.comacuk).

\section{References}

1. (a) Esteve, C.: Vidal, B. Tetrahadron Lett. 2002. 43, 1019. (b) Xiao. Z.: Timberlake. T. W. J. Heterocyclic Chom. 2000. 37.773. (c) Martinez. A.: Gil. C.: Prez. C.: Caastro. A.: Prieto. C.: Otero. J.: Andrei. G.: Snoek. R.: Balzarini. T.: Clerep. E. D. J. Med. Chent. 2000. +3, 3267. (d) Kuang. R: Venkataraman. R: Ruan. S.: Groutas, W. C. Bicong. Hed Chent Lett 1998. 8. 539 . (e) Lee. C.-H.: Kohn. H d. Pham. Sci. 1990. 70. 716. (f) Lee, C.-H: Korp. J. D.: Kohn. H. J. Org. Chem. 1989. 54.3077.

2. (a) Gazieva. G. A.: Kravchenko. A. N.: Lebeder. O. V. Russian Chent. Rev: 2000. 69(3). 221. (b) Aran. V. J.: Goya. P: Ochoa. C. Adances in Heterocwlic Chem. 1988, H.81. (c) MeDermot, S D.; Spillane, W. J. Org. Pre. \& Proc. Int 1984. J6(1), 49.

3. (a) McDermott. S. D. Spillane, W. J. Synthesis 1983. 191. (b) Paquin. A. M. Angew: Chem. 1948. 60. 316.

4. (a) Scott. F. L.: Schaumantl. C. W.: King. J. P. J. Org. Chem. Conmum. 1961. 26.985. (b) Kirsanov. A. V. J. Gen. Chem. 1952. 22. 233 .

5. Lee, C. S.; Kim, S. H.: Lee. C.-H J. Konean Chent. Soc. 1997, 1 . 677. (b) Ohme. R. Schmits. C. Angew: Chem. Int. Ed. Engl 1965 4. 433 .

6. (a) Breining. T.: Cimpoia. A. R.: Mansour. I. S.: Cammack. N.: Hopewell. P: Ashmanl. C. Heterockcles 1995. H. 87. (b) Jadhat. P. K.: Woemer. F. J. Tetrahedron Lett. 1995. 36, 6383. (c) Castro. A.; Martinez. A. J. Chem. Soc., Perkin Trams. 1994. 2. 1561. (d) Groutas. W. C: Kuang. R.: Venkataraman. R. Biochem. Biophys. Res. Conmun. 1994. 198. 341. (e) Schwenker. G.: Guo. H. Arch. Pham. 1993. 326. 45. (f) Abd El Latif. F. M. Asian d. Chem. 1993. 5. 184. (g) Faleschini. G:: Nachbaur. E:: Belaj. F. Phosphorus Sulfir Silicon Relat. Elent. 1992. 65. 147. (h) Alberola, A.: Andres, J. M: Gonwalez, A.: Pedrosa. R: Vicente. M. Sunthesis 1991, 355. (i) Esser, T.: Karu. A. E.: Toia, R. F.: 
Casida, I. E. Chem Res. Toxicol 1991. 4. 162. (j) Alkorta. I: Gova. P.: Paez. A. J. Heteracyctes 1991. 32. 279. (k) Haake. M.: Schummelfelder. B. Sinthesis 1991. 753. (1) Lee, C.-H.: Kohn. H. J. Org. Chem. 1990. 55. 6098. (m) Lee. C.-H.: Kohn. H. J. Heterocyd Chem 1990, 27, 2107. (n) Goyal. R. N.: Bhargava, S. Cum Sci. 1989, 58, 287. (o) Alkorta. I.: Aran, V. J.: Davila. E: Ruiz, I. R.: Stud M. Liebigs Am. Chem 1989,1135 . (p) Lee. C.H.: Kohn. H. Heterocycles 1988. 27. 2581. (q) Aran. V. J.: Goya. P.: Ochoa. C. Att: Heterocyct. Chem. 1988. H. 81. (r) Span. P. 547. 443: Chem. Atostr. 1988. 108. 6031. (s) Alkorta. I: Aran. V. I.: Bielsa. A. G.: Stud M. J. Chem Soc. Perkin Trans. 1989. 1135. (t) Aran. V. J: Ruiz, J. R.: Davila. E.: Alkorta, I.: Stud. M. Liebigs Am. Chem. 1988. 337. (u) Dusemund, J.: Schurreit, T. Awh. Pharm. 1987. 320.534. (v) Dusemund. J: Schurreit. T. Arch. Phomt 1986. 319.826. (w) McDermott. S. D.: Spillane. W J. Org Prep. Proc. Int 1984. 16. 49. (x) Elguero. J.: Ochoa. C.: Stud, M: Esteban-Calderon, C: Martinez-Ripoll, M.: Iayet I. P. Vertut. M. C. J. Org (Chem. 1982, 47. 536. (v) Petersen, H. Symhesis 1973. 243. (z) Lawson. A. Tinkler. R. B. Chem. Ren.
1970. 70.593

7. Muller. G. W.: DuBois. G. E. J. Org Chem. 1989. 54. $447 \mathrm{I}$

8. (a) Lee. J. S.: Yang. I. D.: Kim. S. H.: Ant. S. I.: Lee. C.-H. Bull Korean Chent Soc. 2003. 24(1). 129. (b). Lee. I. S.: Lee. C.-H. Bull. Korean Chent. Soc. 2002, 23(1). 167. (b) Lee, J. S.; Lee C. H. d. Koman Chem Soc. 2001. 45. 92. (c) Kong. Y. J.; Kim, S. H: Lee. C.-H. J. Kowan Chem. Soc. 1999. 13. 131.

9. (a) Appel. R.: Berger. G. Chem Ber 1958. 91. 1339. (b) Grat. R Chent. Ber. 1959. 92. 509. (c) CIBA Ltd. Belgium Patent 640.160. May 19. 1964: Chem. Abstr. 1950. 62. 16134e.

10. (a) Xiac. Z: Timberlake, J. W. J. Heterockel. Chem. 2000, 37. 773. (b) Dewynter. G.: Aouf. N.: Criton. M.: Montero. J. L. Tetrahedron 1993. fo(1),65.

11. Sheldrick. G. M.: Kruger. C. Crystallographic Computing 3: Onford University Press: London. 1985: pp 175-189.

12. Sheldrick. G. M.: Flack. H. D.: Parkanyi. L.: Simon. K Crustallographic Computing 6, Oxford University Press: London. 1993: pp 111-189. 